\title{
Pregnant Women's Perceptions of Patient-Provider Communication for Health Behavior Change during Pregnancy
}

\author{
Jenn Leiferman ${ }^{*}$, Elizabeth Sinatra ${ }^{1}$, Jennifer Huberty ${ }^{2}$ \\ ${ }^{1}$ Department of Community and Behavioral Health, Colorado School of Public Health, Aurora, USA \\ ${ }^{2}$ School of Nutrition and Health Promotion, College of Health Solutions, Arizona State University, Phoenix, USA \\ Email: ${ }^{*}$ ienn.leiferman@ucdenver.edu
}

Received 17 June 2014; revised 15 July 2014; accepted 10 August 2014

Copyright (C) 2014 by authors and Scientific Research Publishing Inc. This work is licensed under the Creative Commons Attribution International License (CC BY). http://creativecommons.org/licenses/by/4.0/ cc) (i) Open Access

\section{Abstract}

Aim: The primary aim of the project was to conduct focus groups with pregnant women to examine their perceptions on patient and health care provider (HCP) communication during prenatal visits pertaining to health behavioral change. In particular, to determine what types of communication facilitate or prevent patient engagement and adherence to certain health behaviors related to smoking cessation, engagement in physical activity, healthy eating and healthy weight gain, and stress management. Methods: Participants were recruited from the obstetric and midwifery clinics at the University of Colorado Hospital. Twenty-four pregnant, English-speaking women between the ages of 18 and 46 years old, the majority of which had full health insurance coverage, participated in one of three focus groups that were conducted. The transcripts were coded for themes and patterns. Results identified numerous current practices of HCPs, facilitators and barriers in care, and patient recommendations related to effective patient-provider communication. Results: Overall many women received basic information about most health behaviors (i.e. healthy eating, physical activity, and smoking cessation) with the exception of stress management from their HCPs via their introductory information packet. However, typically there was no follow-up beyond receipt of the packet. As a result, women sought information online from numerous sources. Unfortunately, this information often conflicted with HCP provided information, as did the information provided from multiple HCPs in group care settings. A major facilitator of behavioral change pertained to building trust and rapport as it directly enhanced the perceived quality of patient-provider communication on prenatal health behaviors. Across all behaviors, women voiced the need for available resources that were credible and referenced by their HCPs. Conclusions: These findings provide a better understanding of what facilitates and prevents

\footnotetext{
${ }^{*}$ Corresponding author.
} 
women from engaging in healthy behaviors during their pregnancy, in addition to improving patient and provider communication.

\author{
Keywords
}

Pregnancy, Prenatal Health, Health Care

\title{
1. Introduction
}

Promoting a healthy lifestyle during pregnancy is essential. There are many behavioral factors associated with a healthy lifestyle such as regular physical activity, proper nutrition and healthy weight gain as well as smoking cessation and stress management. These healthy behaviors affect multiple maternal and child outcomes during the perinatal period. For example, antenatal physical activity is associated with adequate gestational weight gain [1] [2], improved course of delivery [3] [4], reduced incidence of gestational diabetes mellitus [5], preeclampsia [6], and many psychological benefits [7] [8]. A healthy diet and body mass index (BMI) (prior and during pregnancy) can lower the risk of gestational diabetes, preeclampsia, birth defects, intrauterine growth restriction, and later chronic disease (see review by [2]). Maternal smoking can cause premature birth or intrauterine growth restriction resulting in low birth weight and also increase the risk for maternal conditions such as placenta previa, placental abruption, decreased maternal thyroid function and ectopic pregnancy [9]. Lastly, untreated or improperly managed stress can cause serious health conditions through compromising the immune, cardiovascular, neuroendocrine, and central nervous systems leading to adverse birth outcomes such as preterm delivery [10].

Unfortunately many women struggle to engage in healthy prenatal behaviors. For example, pregnant women participate in less physical activity than non-pregnant women and overall physical activity levels decrease as pregnancy progresses [11]. Additionally, approximately 46\% of women gain excessive weight during pregnancy [12] and an estimated $10 \%$ of pregnant women smoke during their last trimester [13]. Pregnancy is an opportune time for women to modify their health behaviors as this transitional time is often thought of as an "external motivator" for behavior change in order to protect the health of the fetus as well as the mother's own health. Providing pregnant women with the necessary knowledge to promote engagement in health behaviors would not only positively impact antenatal health outcomes but also promote positive health outcomes in the postpartum period and beyond. Unfortunately, pregnant women often report a lack of knowledge concerning prenatal health behaviors and often rely on family and friends for advice, resulting in inconsistent messaging. Moreover, pregnant women believe that if they receive information related to how to safely and effectively engage in these prenatal behaviors, such as physical activity, it would facilitate their engagement in them [14].

Healthcare providers can play a significant role in relaying evidence-based prenatal health behavior information to pregnant women. Especially given that a majority of pregnant women report that their healthcare provider has a great influence on their beliefs regarding behavioral change and that brief counseling on behavioral change by healthcare providers increases the likelihood that patients will adopt behaviors [15]. To date, there is a dearth of literature examining the quality of perceived patient-provider communication on prenatal health behaviors. Findings from small qualitative studies that provide patients' perspectives on the quality of patient-provider communication related to healthy weight gain suggest that patients receive mixed messaging, inconsistent information and voice a need for more, consistent advice on healthy weight gain [16] [17].

The primary aim of the present study was to conduct focus groups with pregnant women to examine their perceptions on patient-provider communication pertaining to prenatal health behavioral change. In particular, to determine what types of communication facilitate or prevent patient engagement and adherence to certain health behaviors related to regular physical activity engagement, healthy eating and healthy weight gain, stress management, and smoking cessation.

\section{Methods}

\subsection{Recruitment/Sample}

Prior to conducting this investigation, the study protocol was approved by the Institutional Review Board at the 
respective institution. Women who were pregnant, English speaking and between the ages of 18 and 46 years old were eligible to participate in the focus group. Participants were recruited from local obstetrics and midwifery clinics.

\subsection{Focus Group Procedures}

All participants were guided through informed consent procedures by the study coordinator prior to participating in the focus group. Consent was obtained from each individual and not as a group. Each participant had any questions answered prior to consent. The primary aim of the focus groups was to determine what patient-provider communication factors facilitate or act as barriers to patient health behavioral change during pregnancy. All focus group facilitated discussions were audiotaped. The moderator began with a short welcome statement and overview of the topic. The ground rules, as presented by Krueger and Casey (2000), were read before the questions began [18]. Participants were informed that the focus group sessions were audio recorded, that no individual names would be used to identify their responses, and that the tapes would be destroyed upon completion of the study.

The co-moderator was responsible for taking comprehensive notes, making observations (verbal and nonverbal), operating the tape recorder, and handling the logistics during the focus groups. To ensure internal validity of data collection the co-moderator provided written accounts of the focus groups' proceedings as well [19]. The moderator and co-moderator transcribed both verbal and nonverbal communication exhibited by the participants in the focus group. After each focus group session, a short meeting took place between the moderator, and co-moderator to compare notes, discuss themes, and express areas of improvement, insuring internal validity.

The moderator directed the discussion by asking predetermined, open-ended questions, which were asked in a natural, logical sequence to encourage group discussion. The questions were purposefully intended to be interpreted broadly so that participants were not guided in their responses. For example, "What made it easy to talk with your healthcare provider about healthy eating and healthy weight gain?” More targeted questions were included, however, in the event that participants need more probes. Such as "What made it easy to ask questions and bring up concerns with your HCP?” Each interview took approximately 60 minutes to complete.

\subsection{Qualitative Analytical Methods}

Data were transcribed verbatim and analyzed for themes and patterns. The research team organized data into categories that reflected symbolic domains of meaning, and within domains, relational patterns, and finally overarching themes. First, the lead researcher read each transcript and developed initial categories and overarching themes. Two additional research team members then read through the initial code and then added and refined. The research team read the codes and discrepancies in coding were discussed between the coders until a consensus was achieved. Memos were kept throughout the coding of the data. The synthesis stage of data analysis involved triangulating the findings from FGs, demographic, and health behavior data, exploring comparisons across different subgroups, revisiting the literature to compare findings to other investigations, and answering the research questions as completely as possible. The trustworthiness of study findings were heightened through attention to the credibility, transferability, dependability, and confirmability of the data. Methods to enhance trustworthiness include prolonged engagement, persistent observation, an audit trail, informal member checks, and triangulation. Triangulation involved meetings of the research team to review the range of data, examine contradictory data, and consider the possibility of symbolic meaning or social desirability underlying apparent discrepancies. Memos kept throughout the fieldwork experience documented decisions about sampling, categorization, model formulation, and the like.

\section{Results}

Seventy women were screened of which, 24 participated in a focus group. Please see Figure 1 for recruitment flow chart. The final sample resulted in 24 pregnant, English speaking women between the ages of 18 and 46 years old with the majority being Caucasian $(75 \%$; $n=18)$, 62\% married $(n=15)$ and $75 \%$ having some healthcare insurance $(\mathrm{n}=18)$. Please see Table 1 for descriptive statistics of sample. Three focus groups were conducted with an average of 8 participants per group.

General themes emerged from the data across all health behaviors (e.g., physical activity, healthy eating/weight 


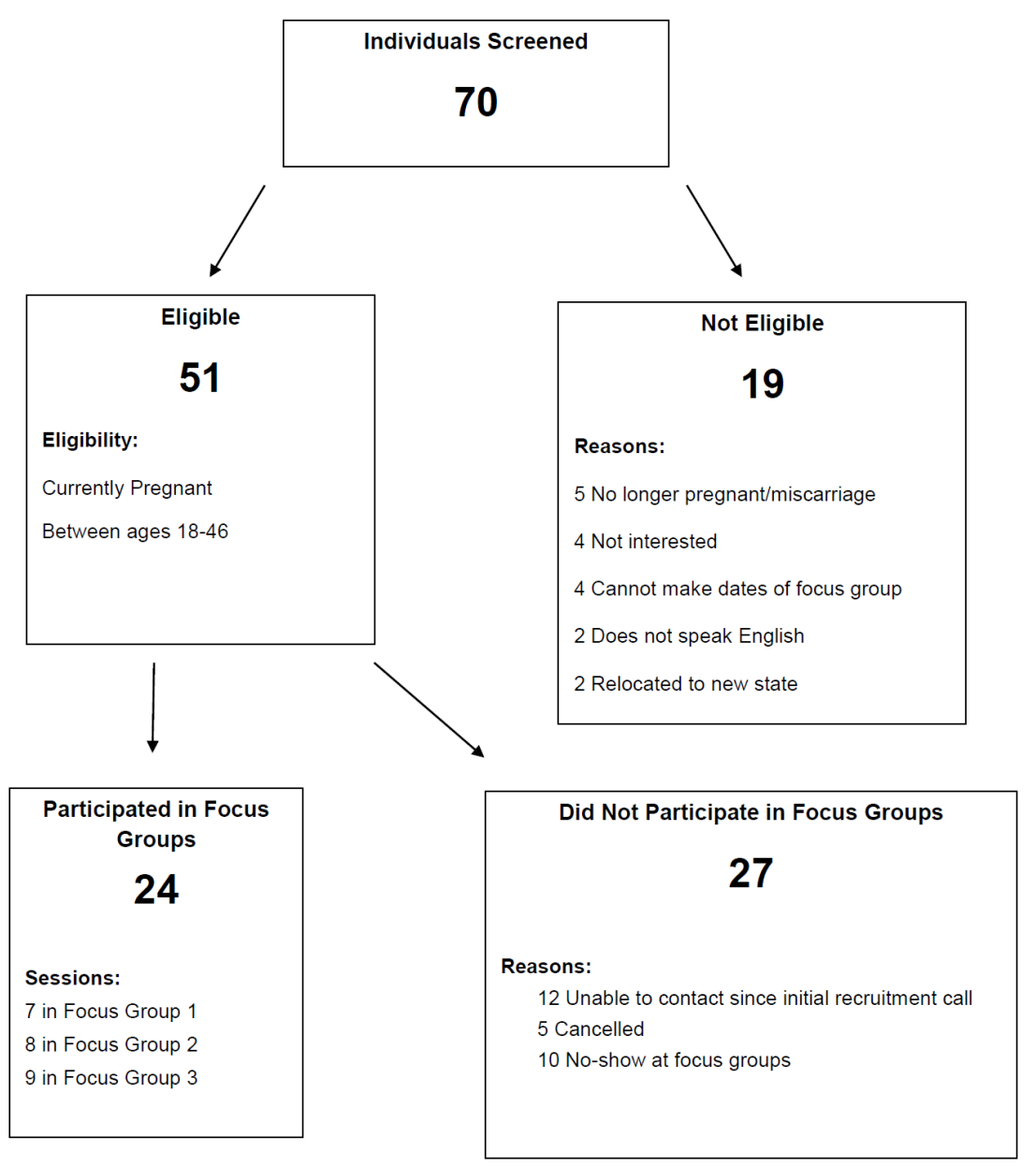

Figure 1. Focus group recruitment flow chart.

Table 1. Descriptive statistics for full sample.

\begin{tabular}{lcc}
\hline \multicolumn{1}{c}{ Variable } & $(\mathbf{N}=\mathbf{2 4})$ & \% \\
\hline Race & 18 & 75 \\
Caucasian & 4 & 16.7 \\
African American & 1 & 4.2 \\
American Indian, Pacific Islander & 1 & 4.2 \\
Other & & \\
Ethnicity & 2 & 8.3 \\
Hispanic/Latino Origin & 22 & 91.7 \\
Non-Hispanic White & & 29.9 \\
Marital status & 7 & 8.3 \\
Single & 2 & 62.5 \\
Divorced or separated & 15 & 70.8 \\
Married & & 4.2 \\
Health coverage & 17 & 25 \\
Health insurance (full coverage) & 1 & 12.5 \\
Health insurance (partial coverage) & 63.3 \\
Medicaid & 6 & 4.2 \\
Education & & \\
$>12$ years & 3 & 1 \\
High school or GED & 20 & \\
College or beyond & & \\
\hline
\end{tabular}


gain, stress management, and smoking cessation). These included current practices, barriers, facilitators, and recommendations. Overall many women stated they received basic information about most health behaviors (i.e. healthy eating, physical activity, and smoking cessation) from their HCPs via their introductory information packet. However, typically there was no follow-up beyond receipt of the packet. As a result, women sought information online from numerous sources. Unfortunately, this information often conflicted with HCP provided information, as did the information provided from multiple HCPs in group care settings. A major facilitator of behavioral change pertained to building trust and rapport as it directly enhanced the perceived quality of patient-provider communication on prenatal health behaviors. However, most women failed to initiate conversation on health behavior unless they had concerns about physical symptomatology or effects on baby. Across all behaviors, women voiced the need for available resources that are credible and referenced by their HCPs. The health behaviors and their themes are presented below.

\section{Physical Activity}

Current practices. Women reported receiving handouts that included information about types of recommended activities (i.e., walking, swimming) but very little information about community resources for activity participation. A few women reported they were given more specific information about physical activity if they asked.

Barriers. The major barrier for physical activity was inconsistent information. Women reported that the information they received from multiple sources (i.e., HCPs, books, online, family, and friends) was inconsistent. Women also reported that they weren't sure of the activities they could engage in safely and therefore felt limited in their options. As a result they chose not to participate in physical activity. One woman said, "Unfortunately, there is a lot of you can't really tell what you specifically can do or can't do until you do something too much.. And so that's then frustrating."

Facilitators. Women reported that when they received consistent messaging, this helped them to be physically active. This was especially true when the information was related to current and previous physical activity levels. Women reported initiating conversation with their HCPs when they were experiencing discomfort and/or concerned about safety. One woman said, "I actually brought it (exercise) up because of experiencing discomfort while I was exercising and I feel like I really struggled with getting good answers on what are the limitations. I feel like I'm listening to my body but when I'm done, I don't feel so good”. Women reported being more likely to initiate this conversation if they had "mixed messages" from information they received and would seek advice from their HCPs to clarify the mixed messaging. "I would prefer to just default to my physician" one woman said. Women shared that their HCPs were more likely to talk about physical activity when they talked about the safety of the baby. Women also expressed that they were more likely to be active if they were given clearer "black and white" advice as compared to "just listen to your body." Unfortunately, most of the HCP advice given conveyed the latter.

Recommendations. The women provided numerous recommendations related to how HCPs could improve their communication about physical activity. Women thought that HCPs should provide information related to physical activity in paper form - something they could take home and refer to at a future time point. More specifically they preferred to have evidence-based, current guidelines for physical activity. "I definitely think more information would have helped me be more physically active. If I knew more, I think I would have done more... because the information they (HCPs) gave was so general and vague and it just wasn't worth it”. Women also shared that they would like their HCPs to be consistent with their messaging and emphasize the safety of physical activity. Interestingly, women wanted to be held accountable and thought their HCP could help them stay motivated by helping them to set goals and hold them accountable for physical activity participation. "If my doctor was pushing it (exercise) because of health concerns or reasons then I would definitely carve out more time for it but if he doesn't see the problem with, you know, my weight gain or anything or doesn't see fitness as something to help whatever else is going on than I wouldn't change what I do”. Finally, women wanted reputable resources for more information related to physical activity.

\section{Healthy Eating/Healthy Weight Gain}

Current practices. Most women received an informational package containing limited information on nutrition at their initial visit. A few practices also covered weight gain at the initial visit. "At my first visit, they went through what would be the ideal weight gain and how I should progress during the pregnancy but that was the 
most covered about nutrition and weight gain.”

Barriers. Women felt that they either had limited information provided to them about healthy eating or inconsistent messaging. Despite this, women were not concerned about their eating or weight gain for a number of reasons. First, they reported that they didn't really need nutrition information from their HCP because there are a number of other sources in which they could access the information. Second the HCP didn't seem to be concerned with healthy eating (or never talked about it) unless there was an issue with weight and weight gain was primarily assessed and shared at first visit only.

One woman said, "At my first appointment they told me how much they would like me to gain during the pregnancy. ... didn't really focus on nutrition at all. It was just... weight gain was the overall thing. I didn't see that they wanted that to be from healthy stuff like fruits and vegetables, or making sure I got enough-protein, or things like that. It was just the overall weight—gain. It was... I kinda left feeling like, ok if I just ate- Twinkies, but only gained that amount of weight they'd be ok with it."

Women assumed that everything was ok if the HCP didn’t discuss their dietary behaviors, “... so I feel like if there was too much weight gain, or not enough that would be brought up in the appointment, or "they weigh you and never mention anything to me, you know, I thought ok everything must be ok otherwise they’d bring it up. I just feel like there's just a lot of trust there or unspoken trust like we get weighed every time... if there is a concern then my provider is looking out for any red flags.” Finally, women shared that they "trusted their body-if I am craving it I must need it”.

Facilitators. Women thought that having basic information about healthy eating, primarily about which foods to avoid, and on fruit and vegetable intake helped their eating behaviors. A few women said that they were given nice packets with ideas of snacks and types of foods through the WIC program and that midwives were more likely to be giving feedback on which types of foods to consume. Women also reported that having weight gain ranges at their first visit (e.g., percentiles) and weight gain monitored at every appointment facilitated weight gain. Some women who had special conditions such as anemia or gestational diabetes found having information tailored for their condition (e.g., what is healthy weight gain if I have gestational diabetes?) was very helpful. Because few HCPs were providing information about healthy eating during appointments, most women were getting information online to help facilitate their healthy eating and healthy weight gain. Only a few women stated that they asked their HCP for eating information and this was for reasons related to symptomology, such as cramps or morning sickness. Some women shared they were monitoring their water intake and others were more concerned with quantity of calories as compared to quality but their HCPs were providing little direction related to these topics.

One woman said, "I feel just like-all of it, you know, like what foods you should be eating, how many calories you should be taking in, what are the portions for the carbs, fat, protein, and then also I like the idea of what you — can't eat. ..., but just having that knowledge... and feeling empowered to be like ok well this is ok to do, or-not ok-stay away from it, so just—all of it would be helpful."

Recommended practices. Women made many suggestions for future practice related to healthy eating and healthy weight gain. Women thought that weight gain should be charted throughout the pregnancy (i.e., beginning, middle, and end) and discussed with the patient by the HCP. They also thought that women should know what their weight gain is in relation to the baby's weight gain. Similarly to the recommendations for physical activity, women wanted assistance with self-monitoring, "What about if you self-monitor a little bit because now I have no clue what's right, wrong, good, bad, but if there is self-monitoring involved and having someone else help you," would be helpful.

Women thought HCPs should ask them to "jot down food” or keep a food diary. Women also shared that their eating should be targeted to their own individual symptoms (e.g., morning sickness, fatigue). Importantly, women thought that they should have a meeting with their HCP (or nutritionist) to obtain healthy eating and weight information for more than five minutes of time. Specifically they wanted this appointment to be once earlier in the pregnancy and once in the middle, or once during each trimester. Women also suggested providing what types of foods to eat and ways to prepare them (e.g., healthy recipes), creative combinations of foods, and a list of foods to avoid.

\section{Stress Management}

Current practices. Overall, practices for stress management were minimal and mostly initiated by the patient as 
compared to the HCP. Women that reported being screened for depression and a few for stress said that they were asked close-ended questions and there was little to no follow-up by the HCP. "I can't even recall being asked about stress management". "There was a few weeks where I was really stressed and I was in here a couple of times for pre-labor and stuff like that just you know, I'm really stressed but they didn't really address it with stress management they were like ok your stressed, but they didn't help me with that".

One woman said that only pamphlets were provided to her with minimal follow-up, "My last pregnancy they just handed me like 6 different pamphlets on my way out the door and were like "if you get postpartum depression, here you go" and so I don't know if they just don't do it during doctor's appointments because they figure they could just do it at discharge or what?" As a result women said they used the Internet, specifically chat rooms to discuss mental health concerns. Some women mentioned that once they brought their stress to the attention of their HCP, they received follow-up information (e.g., packet of resources about what to do, how to handle stress, phone-number to call). A few reported that they screened positive for depression early in their pregnancy and that their HCPs followed-up with conversations at subsequent visits. One woman shared, "Well, I was very stressed when I first found out I was pregnant. So there was talk, ... if I went for a walk everyday, everything would just be better in my life, which, you know if I'm stressed about going out for a walk everyday you know that's only adding to my stress. But they also talked about resources either that they had; psychiatrists you can talk to, numbers you can call 24-hours a day if you're feeling depression or stress or anxiety. And so they were very informative, ... if they've asked, at every appointment it's been you know, "how are you feeling?" "how is your stress level." A few others mentioned that their HCP mentioned yoga or massage for stress and/or were routinely asked if they were feeling depressed.

Barriers. Even though HCPs may acknowledge stress, HCPs often failed to provide guidance on next steps of dealing with stress. Women reported that they only brought up stress if they were worried it would affect the baby or the pregnancy or if it would be taken the wrong way or give them some sort of stigma that is associated with women who have depression. A woman said, "If you just feel stressed they'll think you're depressed. ... so you can't really say anything... to certain people because they take it the wrong way.” Women also mentioned that they thought their stress was common and that it "just happens", is "normal” and as such, wasn't even "on their radar". Some women did mention that they felt rushed in their appointments and didn't have enough time to open up about their stress. Interestingly women thought there was a plethora of information available to them through other sources related to stress management and this actually contributed to the stress they were feeling. One woman shared, "I think I went through the same thing where I got a little information crazy because there is so much stuff out there, and then I just ended up getting really anxious".

Facilitators. To help women manage their stress, women wanted to develop a more personal relationship with their HCP. Women reported that when they felt they had developed a strong relationship with their HCP, they were more likely to talk to the HCP about stress. The relationship was further cultivated if they were able to see the same HCP (helped them to develop trust) and if the HCP really listened to their concerns. One woman said, "I (HCP) am here for you." ... "they just don't, in my experience, talk like that and I think if somebody was like that I would be like, oh wow you actually care.” Women also reported that when their HCP was there for major life events and adequate resources were available they were more likely to manage their stress. Women also said that they would be more likely to manage stress if the HCP linked their stress to the health of the baby. "I would only bring it up with my provider if I felt like it was affecting my baby or my pregnancy negatively then I would bring it up but if I was just feeling stressed than I would be talking to my family like I wouldn't feel like I need to tell my providers about it”. Some women said that handouts on yoga and internet sources were helpful in reducing stress but it was also helpful to have strategies manage or cope with perceived stressors.

Recommendations. Women suggested that for stress management, HCPs should provide more information so they know what their options are and have some support, "I would have liked to known about the clinic (perinatal mood disorder clinic affiliated with a local obstetric clinic). I would have liked to known it was available or it was an option if I was feeling stressed out...”. Women especially wanted support from other moms through support calls or message boards, "just someone you can talk to". They also would like HCPs to offer information about what is available in their community such as yoga, massage and exercise classes to help with stress management. Similar to the facilitators for stress management, women recommended that HCPs develop relationships with their patients, if possible enhance continuity of care by seeing the same patients (rather than someone else in their group practice) and really reflectively listen to the patient. 


\section{Smoking}

Current practices. Although smoking was not discussed as much as the other health behaviors (in part because most of the women were not smokers), women shared that their HCPs asked about smoking cessation typically in the paperwork during their initial pregnancy appointment. Patients also received information on smoking cessation in their initial visit information packet. One woman said, “As soon as they made sure that I didn't smoke and no one in the house (did) it's never been questioned since." Unfortunately, little information was provided on the harmful effects of secondhand smoking.

Barriers. Interestingly women mentioned that their smoking habits may be too embarrassing to discuss. Women also mentioned that if they weren't smoking than they were less likely to discuss the hazards of secondhand smoke.

Recommendations. Women recommended that they be provided information about dangers of second-hand smoke and preferred this come from their HCPs as opposed to finding it on the web. "It would be good if they (HCPs) went through because they are professionals and let them pick and choose you know what are the good sites. What are the sites that give information that we as a practice back up".

Additional communication barriers across all behaviors. There were also come system level barriers related to group care settings. Often times seeing multiple providers resulted in inconsistent messaging and lack of continuity of care. "That's the one think I don't like about big practices as far as most of them are multiple providers and very rarely see the same ones and even small practices you see many different people and I just think that sometimes it (information on prenatal health behaviors) gets lost. They forget to make a note and then the next person (HCP) has to follow up or they forget you know". Group care was also reported to feel "impersonal". "But I just go to the same like 6 or 7 different midwives so it feels really impersonal, they ask me questions the same questions over and over".

There were also some overall HCP qualities that were associated with perceived overall effective quality of patient-provider communication such as not feeling rushed. "They never make me feel rushed when I come to an appointment. They always make me feel like I have the time to ask all of my questions no matter how big or small or stupid it might be”. Feeling supported and overcoming the note-taking distractions also were also perceived positively. "I think my biggest two things that I looked for and kind of appreciated from my HCP was being able to ask questions and also feeling supported with my decisions. "I think you can tell when somebody is concerned and actually listening". "And I think it's an actual skill, but some people are much better about either talking to you the whole time or having a good routine of typing while they talk to you but still acknowledging you. And some people just seem to be overwhelmed by the computer piece."

\section{Discussion}

The primary aim of the present study was to determine pregnant women's perceptions about patient-provider communication pertaining to prenatal health behavior change. In particular, to determine what types of communication facilitates or prevents patient engagement and adherence to certain health behaviors related to regular physical activity engagement, healthy eating and healthy weight gain, stress management, and smoking cessation. Our findings suggest that pregnant women receive limited basic information on prenatal health behaviors, typically in the form of a paper packet during their initial visit. Patients often perceive this information to be inconsistent and inadequate and express a need for consistent messaging from reputable resources. Additionally, women would prefer this information and resources be provided by their HCP as they view the delivery of information from their HCP most influential.

The Department of Health and Human Services (DHHS) and American Congress of Obstetricians and Gynecologists (ACOG) recommend that pregnant women free of obstetric complications engage in moderate-intensity physical activity on all or most days of the week [20] [21]. Healthcare providers can play a significant role in relaying correct information regarding prenatal physical activity to their patients. Unfortunately, close to $50 \%$ of HCPs are not aware of the recommended guidelines for physical activity [22] or fail to discuss the guidelines with their patients [23] [24]. This is consistent with our findings that many pregnant women reported receiving little information on physical activity from their HCPs and if they did the information was often inconsistent.

Healthy eating and adequate weight gain are important during pregnancy as they reduce the risk of numerous poor perinatal outcomes such as gestational diabetes, preeclampsia, cesarean delivery, weight retention after delivery, adverse birth outcomes, and higher rates of unsuccessful breastfeeding [25] [26]. Pregnant women, who 
stay within the range of recommended weight gain (recommendation varies based on their pre-pregnancy Body Mass Index) are better able to return to a healthy weight postpartum. Unfortunately, approximately $46 \%$ of women 20 years or older who were pregnant reported excessive gestational weight gain.

Often excessive weight gain and a poor diet are due to a lack of knowledge and skills related to healthy eating. Prenatal checkups provide clinicians with an opportunity to advise women about healthy weight gain throughout pregnancy and postpartum. The Institute of Medicine (IOM) provides guidelines on appropriate weight gain throughout the pregnancy based on a woman's pre-pregnancy BMI. These IOM recommendations for gestational weight gain are based on pre-pregnancy BMI with the recommended weight gain as follows (underweight: 28 - 40 lbs; normal weight 15 - 25 lbs; overweight 15 - 25 lbs, and obese at least 15 lbs). HCPs play an integral role in encouraging and promoting healthy nutrition and weight gain by serving as a critical source for knowledge, skill development, and motivation for their patients. Moreover, appropriate gestational weight gain is a topic clinicians need to be aware of in-order to provide optimal obstetric care.

Our findings suggest that most HCPs provide very little follow-up on healthy eating and weight gain beyond the initial informative packet and the obtainment of individual weight at each prenatal visit. This is consistent with others who have reported that patients' perceived HCPs didn't discuss or seem to care too much about gestational weight gain and voiced a need for more information and advice on weight gain [16] [17]. Phelan et al. found that less than half of patients reported receiving information or advice on weight gain from their HCPs [1]. Recent research suggests that there is a disconnect between patient-provider perspectives on communication pertaining to healthy eating and physical activity. Such that, HCPs report they talk about nutrition and physical activity with all patients whereas patients report that their HCPs either talked in general terms or not at all [27].

Interestingly, women in our study, who were considered of "higher risk" for obstetric complications or asked specific questions related to weight and nutrition tended to report receiving more guidance on weight gain and associated behaviors. Consistent with our findings, Duthie et al., (2013) reported patients perceived little receipt of "unsolicited advice" but did receive more information about healthy weight gain if the patient asked specific questions about it or related healthy eating and physical activity behaviors [27]. In our study, proactive patients felt they received more information from HCPs but they still were not completely satisfied with advice/care received. Healthy nutrition and weight should be established prior to conception and throughout the prenatal period in order to optimize maternal and child health.

Pregnancy is an exciting stage in a woman's life, but is often accompanied by unpleasant symptoms and stressors such as physical changes in the body, feeling nauseous or more tired than usual, having physical ailments or mood changes, having concerns or worries about labor, delivery, the baby's health, or parenting, and having stress within relationships. These emotional, hormonal, and physical changes, in addition to other stresses in life spanning from daily hassles (e.g. domestic affairs, financial issues) to major life events or crises (e.g. divorce, illness or death of someone close), can result in severe health problems, conditions, and negative birth outcomes.

There is also a link between stress and weight gain during the prenatal period with a significant dose-response relationship between stressful life events and gestational weight gain, especially in early pregnancy [28]. In addition, stress during pregnancy is associated with unhealthy eating and lack of physical activity [29]. Therefore, techniques and strategies to aid in managing stress are critical to incorporate during prenatal care to improve the health and wellbeing of both mother and baby. According to the recent PRAMS data, 70\% of women have experienced at least three stressors and $21 \%$ have experienced more than six stressors during their pregnancy (www.colorado.gov). Although stress management techniques can aid in managing everyday stressors, untreated or improperly managed stress can cause serious health conditions through compromising the immune, cardiovascular, neuroendocrine, and central nervous systems.

ACOG's latest Committee Opinion advocates for assessing psychosocial risks (e.g. stress and smoking) at least once per trimester as well as helping women manage psychosocial stressors [30]. When necessary HCPs should refer patients for additional evaluation and/or intervention as needed. Our findings suggest these recommendations are not fully being met with the majority of patients reporting little to no assessment of psychosocial stress and a lack of follow up involving guidance to manage stressors and seek further evaluation/intervention. Some patients reported assessment for depression but the majority reported little to no assessment of psychosocial stress and a lack of follow up involving guidance to manage stressors and seek further evaluation/intervenetion. Clearly there is a need for improvement in this area.

Over twenty percent of low birth weight babies could be prevented by cessation of smoking during pregnancy 
[31]. Smoking cessation before 15 weeks gestation yields the greatest health benefit for pregnant women and their unborn child. However, quitting anytime is still beneficial. Offering women services as early as possible in pregnancy is recommended for interventions to be most effective. Consistent with previous research [13], women in our study reported tobacco use was assessed by their HCPs and for those who disclosed use further evaluation and intervention were provided. Given this, better understanding HCP training on smoking cessation and how this influences their knowledge and attitudes related to providing guidance on smoking cessation may be informative in enhancing HCP guidance on other prenatal health behaviors such as healthy eating, physical activity and stress management.

Across all prenatal behaviors a major facilitator of behavioral change pertained to perceived quality of the patient-provider communication—a key aspect of the provider-patient relationship [32]. In particular, relationships that exhibited key components of effective communication such as building trust and rapport were commonly mentioned as facilitators to behavioral change. This is not surprising given effective patient-provider communication during the prenatal period is often characterized by having an empathic conversational style, openness to questions, and allowing sufficient time to have questions answered [33].

A major barrier for providing guidance on all prenatal health behaviors was highlighted pertaining to the lack of continuity of care and how it impedes effective follow-up on prenatal behavioral change. Many women voiced the desire for more comprehensive care by a consistent provider. This finding is confirmed with a recent meta-analysis suggesting that women want comprehensive care with relaxed visit time, consistent providers and to be more active in their own care [34].

Participants offered various recommendations to improve patient-provider communication on prenatal health behaviors. In general, building a solid patient-provider relationship by establishing solid communication involving building trust and rapport, reflective listening, and empathy was stressed. Women also recommended HCPs provide consistent, concrete guidelines to promote prenatal health behaviors and initiate discussion on importance of prenatal health behaviors. Ideally, women would like HCPs to be more active in patient goal setting and plans for behavioral change. There was a definite need voiced for a central resource (recommended by HCP) of evidence-based resources offering different option to help facilitate prenatal health behaviors. Women currently look to the internet for much of their information gathering on prenatal health behaviors [35], underscoring the importance of having the HCP provide evidence-based information and resources to reduce the uncertainty and anxiety often experienced from sifting through the masses of resources and mixed messages found on the internet.

Promoting engagement in health behaviors during pregnancy is essential. However, many women fail to engage in healthy lifestyles during pregnancy and often times it is due to lack of knowledge and skills. Healthcare providers (HCPs) play an integral role in promoting healthy lifestyle behaviors during pregnancy. Especially given that the majority of pregnant women indicate that their HCP has great influence on their beliefs regarding behavioral change and that brief counseling on behavioral change by HCPs increases the likelihood that patients will adopt behavior [15] [36]. Thus, HCPs are a knowledgeable source of information for women, particularly during a major life transition such as pregnancy.

The majority of HCPs correctly believe that engagement in prenatal health behaviors such as regular prenatal physical activity and healthy nutrition are associated with numerous positive maternal and child outcomes. However, many HCPs are unaware of the national guidelines for these behaviors [23]. Moreover, patients often report information obtained is inconsistent or completely lacking. Clearly, there is a need to enhance HCP knowledge and skill building related to better communication with patients on providing guidance on prenatal health behaviors.

\section{Limitations}

This study has a few limitations. First, there is limited generalizability of the results since the small sample is limited to a few clinics and thus is not representative of all US pregnant women. It is possible that more guidance on prenatal health behaviors may have been given with other healthcare staff that was not captured in this data collection. Patient responses may be affected by recall bias.

\section{Conclusion}

These findings underscore the missed opportunity to use prenatal visits to promote a healthy lifestyle in the pe- 
rinatal period and beyond. Lack of adequate HCP training may be partly responsible as many HCPs report feeling inadequately trained or confused about how to counsel women on health behavior change, especially related to weight management [37] and behaviors associated with it, such as physical activity and healthy eating [23]. Moreover, when it comes to providing guidance on antenatal weight gain, HCPs may not always follow IOM guidelines [37]. It would be remiss to not acknowledge the constraints on the current healthcare system and the limited time allotted per patient visit. Given this, attractive options to offset these inherent limitations and help supplement intervention beyond the HCPs guidance should be highlighted. First providing referral to reputable, evidence-based health education services (e.g. Text 4 Baby) may help to assure that women are getting valuable information and may contribute to their participation in healthier behaviors [35]. Second, HCPs could encourage of use of tracking tools (e.g. FitBit, Jawbone Up, Nike Fuel) to help patients self-monitor behaviors and reach goals. Finally, having other clinical staff in addition to the primary obstetric HCP provides further intervention when necessary. HCPs represent a valuable and influential source to help pregnant women engage in health behaviors. More research in this area is warranted.

\section{Acknowledgements}

The research team would like to acknowledge Amber Chandler for her help with data collection.

\section{Competing Interests}

The authors declare no competing interests.

\section{Author's Contributions}

JL conceived of the study, design, implementation and assessment as well as participated in all stages of study and manuscript preparation. ES contributed to data collection, analysis, and preparation of manuscript. JH contributed to interpretation of results and preparation of manuscript.

\section{Funding}

Work Supported by the March of Dimes.

\section{References}

[1] Phelan, S., Phipps, M.G., Abrams, B., Darroch, F., Schaffner, A. and Wing, R.R. (2011) Randomized Trial of a Behavioral Intervention to Prevent Excessive Gestational Weight Gain: The FIT for Delivery Study. American Journal of Clinical Nutrition, 93, 772-779. http://dx.doi.org/10.3945/ajcn.110.005306

[2] Streuling, I., Beyerlein, A., Rosenfeld, E., Hofmann, H., Schulz, T. and von Kries, R. (2011) Physical Activity and Gestational Weight Gain: A Meta-Analysis of Intervention Trials. BJOG, 118, 278-284. http://dx.doi.org/10.1111/j.1471-0528.2010.02801.x

[3] Clapp, J.F. (1990) The Course of Labor after Endurance Exercise during Pregnancy. American Journal of Obstetrics Gynecology, 163, 1799-1805. http://dx.doi.org/10.1016/0002-9378(90)90753-T

[4] Misra, D.P., Strobino, D.M., Stashinko, E.E., Nagey, D.A. and Nanda, J. (1998) Effects of Physical Activity on Preterm Birth. American Journal of Epidemiology, 147, 628-635. http://dx.doi.org/10.1093/oxfordjournals.aje.a009503

[5] Tobias, D.K., Zhang, C., ven Dam, R.M., Bowers, K. and Hu, F.B. (2011) Physical Activity before and during Pregnancy and Risk of Gestational Diabetes Mellitus: A Meta-Analysis. Diabetes Care, 34, 223-229.

[6] Sorensen, T.K., Williams, M.A., Lee, I., Dashow, E.E., Thompson, M.L. and Luthy, D.A. (2003) Recreational Physical Activity during Pregnancy and Risk of Preeclampsia. Hypertension, 41, 1273-1280. http://dx.doi.org/10.1161/01.HYP.0000072270.82815.91

[7] Poudevigne, M.S. and O’Connor, P.J. (2006) A Review of Physical Activity Patterns in Pregnancy Women and Their Relationship to Psychological Health. SportsMED, 36, 19-38. http://dx.doi.org/10.2165/00007256-200636010-00003

[8] Gaston, A. and Cramp, A. (2011) Exercise during Pregnancy: A Review of Patterns and Determinants. Journal of Science and Medicine in Sport, 14, 299-305. http://dx.doi.org/10.1016/j.jsams.2011.02.006

[9] USDHHS (2010) How Tobacco Smoke Causes Disease: The Biology and Behavioral Basis for Smoking-Attributable Disease. http://www.surgeongeneral.gov/library/reports/smokingconsequences/index.html

[10] Coussons-Read, M.E., Okun, M.L. and Nettles, C.D. (2007) Psychosocial Stress Increases Inflammatory Markers and 
Alters Cytokine Production across Pregnancy. Brain, Behavior, and Immunity, 21, 343-250. http://dx.doi.org/10.1016/j.bbi.2006.08.006

[11] Zhang, J. and Savitz, D.A. (1996) Exercise during Pregnancy among US Women. Annals of Epidemiology, 6, 53-59. http://dx.doi.org/10.1016/1047-2797(95)00093-3

[12] Olson, C.M. (2008) Achieving a Healthy Weight Gain during Pregnancy. Annual Review of Nutrition, 28, $411-423$. http://dx.doi.org/10.1146/annurev.nutr.28.061807.155322

[13] CDC (2013) Smoking Ref Website. http://www.cdc.gov/Reproductivehealth/TobaccoUsePregnancy/index.htm

[14] Kieffer, E.C., Willis, S.K., Arellano, N. and Guzman, R. (2002) Perspectives of Pregnant and Postpartum Latino Women on Diabetes, Physical Activity and Health. Health Education \& Behavior, 29, 542-556. http://dx.doi.org/10.1177/109019802237023

[15] Krans, E.E., Gearhart, J.G., Dubbert, P.M., Klar, P.M., Miller, A.L. and Replogle, W.H. (2005) Pregnant Women’s Beliefs and Influences Regarding Exercise during Pregnancy. Journal of the Mississippi State Medical Association, 46, 67-73.

[16] Furness, P.J., McSeveny, K., Adren, M.A., Garland, C., Dearden, A.M. and Soltani, H. (2011) Maternal Obesity Support Services: A Qualitative Study of the Perspectives of Women and Midwives. BMC Pregnancy and Childbirth, 11, 69.

[17] Stengel, M.R., Kraschnewski, J.L., Hwang, K. and Chuang, C.H. (2012) “What My Doctor Didn’t Tell Me”: Examining the Healthcare Provider Advice to Overweight and Obese Pregnant Women on Gestational Weight Gain and Physical Activity. Women's Health Issues, 22, e535-e540. http://dx.doi.org/10.1016/j.whi.2012.09.004

[18] Krueger, R.A. and Casey, M.A. (2000) A Practical Guide for Applied Research. Sage Publications, Inc., California.

[19] McDaniel, R.W. and Bach, C.A. (1994) Focus Groups: A Data Gathering Strategy for Nursing Research. Nursing Science Quarterly, 7, 4-5. http://dx.doi.org/10.1177/089431849400700103

[20] US Department of Health and Human Services (2008) Physical Activity Guidelines for Americans. Services. US Department of Health and Human Services, Washington DC.

[21] ACOG (2002) Committee Opinion \#267: Exercise during Pregnancy and Postpartum Period. Obstetrics \& Gynecology, 99, 171-173. http://dx.doi.org/10.1016/S0029-7844(01)01749-5

[22] Carroll, J.K., Antognoli, E. and Flocke, S.A. (2011) Evaluation of Physical Activity Counseling in Primary Care Using Direct Observation of the 5As. Annals of Family Medicine, 9, 416-422. http://dx.doi.org/10.1370/afm.1299

[23] Leiferman, J.A., Gutilla, M., Paulson, J.F. and Pivarnik, J. (2012) Antenatal Physical Activity Counseling among Healthcare Providers. Open Journal of Obstetrics and Gynecology, 2, 346-355. http://dx.doi.org/10.4236/ojog.2012.24073

[24] Bauer, P.W., Broman, C.L. and Pivarnik, J.M. (2004) Exercise and Pregnancy Survey for Health Care Providers. MSSE, 36, S113.

[25] Johnson, J., Clifton, R.G., Roberts, J.M., Myatt, L., Hauth, J.C., Spong, C.Y., et al. (2013) Pregnancy Outcomes with Weight Gain above or below the 2009 Institute of Medicine Guidelines. Obstetrics \& Gynecology, 121, 969-975. http://dx.doi.org/10.1097/AOG.0b013e31828aea03

[26] Nohr, E.A., Vaeth, M., Baker, J.L., Sorenson, T.I.A., Olsen, J. and Rasmussen, K.M. (2008) Combined Associations of Pre-Pregnancy Body Mass Index and Gestational Weight Gain with the Outcome of Pregnancy. American Journal of Clinical Nutrition, 87, 1750-1759.

[27] Duthie, E.A., Drew, E.M. and Flynn, K.E. (2013) Patient-Provider Communication about Gestational Weight Gain among Nulliparous Women: A Qualitative Study of the View of Obstetricians and First-Time Pregnant Women. BMC Pregnancy and Childbirth, 13, 231-240. http://dx.doi.org/10.1186/1471-2393-13-231

[28] Zhu, P., Huang, W., Hao, J.H., Huang, K., Jiang, X.M. and Tao, F.B. (2013) Time-Specific Effect of Prenatal Stressful Events on Gestational Weight Gain. International Journal of Gynecology and Obstetrics, 122, 207-211. http://dx.doi.org/10.1016/j.ijgo.2013.04.007

[29] Lobel, M., Cannella, D.L., Graham, J.E., DeVincent, C., Schneider, J. and Meyer, B.A. (2008) Pregnancy-Specific Stress, Prenatal Health Behaviors, and Birth Outcomes. Health Psychology, 27, 604-615. http://dx.doi.org/10.1037/a0013242

[30] ACOG (2006) ACOG Committee Opinion No 343: Psychosocial Risk Factors: Perinatal Screening and Intervention. Obstetrics and Gynecology, 108, 468-477.

[31] Fiore, M.C., Baker, T.B., et al. (2008) Treating Tobacco Use and Dependence.

[32] Emanuel, E.J. and Emanuel, L.L. (1992) Four Models of the Physician-Patient Relationship. JAMA, 267, $2221-2226$. http://dx.doi.org/10.1001/jama.1992.03480160079038 
[33] Raine, R., Cartwright, M., Richens, Y., Mahamed, Z. and Smith, D. (2010) A Qualitative Study of Women’s Experiences of Communication in Antenatal Care: Identifying Areas for Action. Maternal and Child Health Journal, 14, 590-599. http://dx.doi.org/10.1007/s10995-009-0489-7

[34] Novick, G. (2009) Women's Experience of Prenatal Care: An Integrative Review. Journal of Midwifery \& Women’s Health, 54, 226-237. http://dx.doi.org/10.1016/j.jmwh.2009.02.003

[35] Huberty, J., Dinkel, D., Beets, M.W. and Coleman, J. (2013) Describing the Use of Internet for Health, Physical Activity, and Nutrition Information in Pregnant Women. Maternal and Child Health Journal, 17, 1363-1372. http://dx.doi.org/10.1007/s10995-012-1160-2

[36] Aittasalo, M., Pasanen, M., Fogelholm, M., Kinnunen, T.I., Ojala, K. and Luoto, R. (2008) Physical Activity Counseling in Maternity and Child Health Care-A Controlled Trial. BMC Women's Health, 8, 14. http://dx.doi.org/10.1186/1472-6874-8-14

[37] Stotland, N.E., Gilbert, P., Bogetz, A., Harper, C.C., Abrams, B. and Gerbert, B. (2010) Preventing Excessive Weight Gain in Pregnancy: How Do Prenatal Care Providers Approach Counseling? Journal of Women's Health, 19, 807-814. http://dx.doi.org/10.1089/jwh.2009.1462 
Scientific Research Publishing (SCIRP) is one of the largest Open Access journal publishers. It is currently publishing more than 200 open access, online, peer-reviewed journals covering a wide range of academic disciplines. SCIRP serves the worldwide academic communities and contributes to the progress and application of science with its publication.

Other selected journals from SCIRP are listed as below. Submit your manuscript to us via either submit@scirp.org or Online Submission Portal.
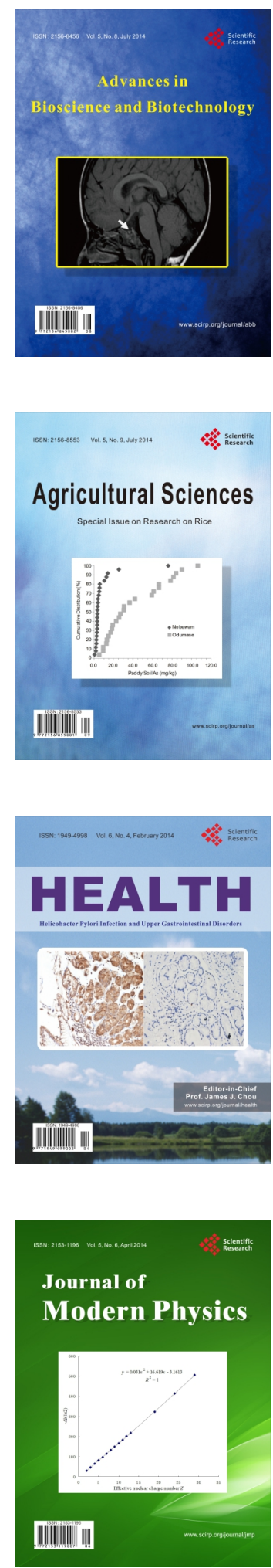
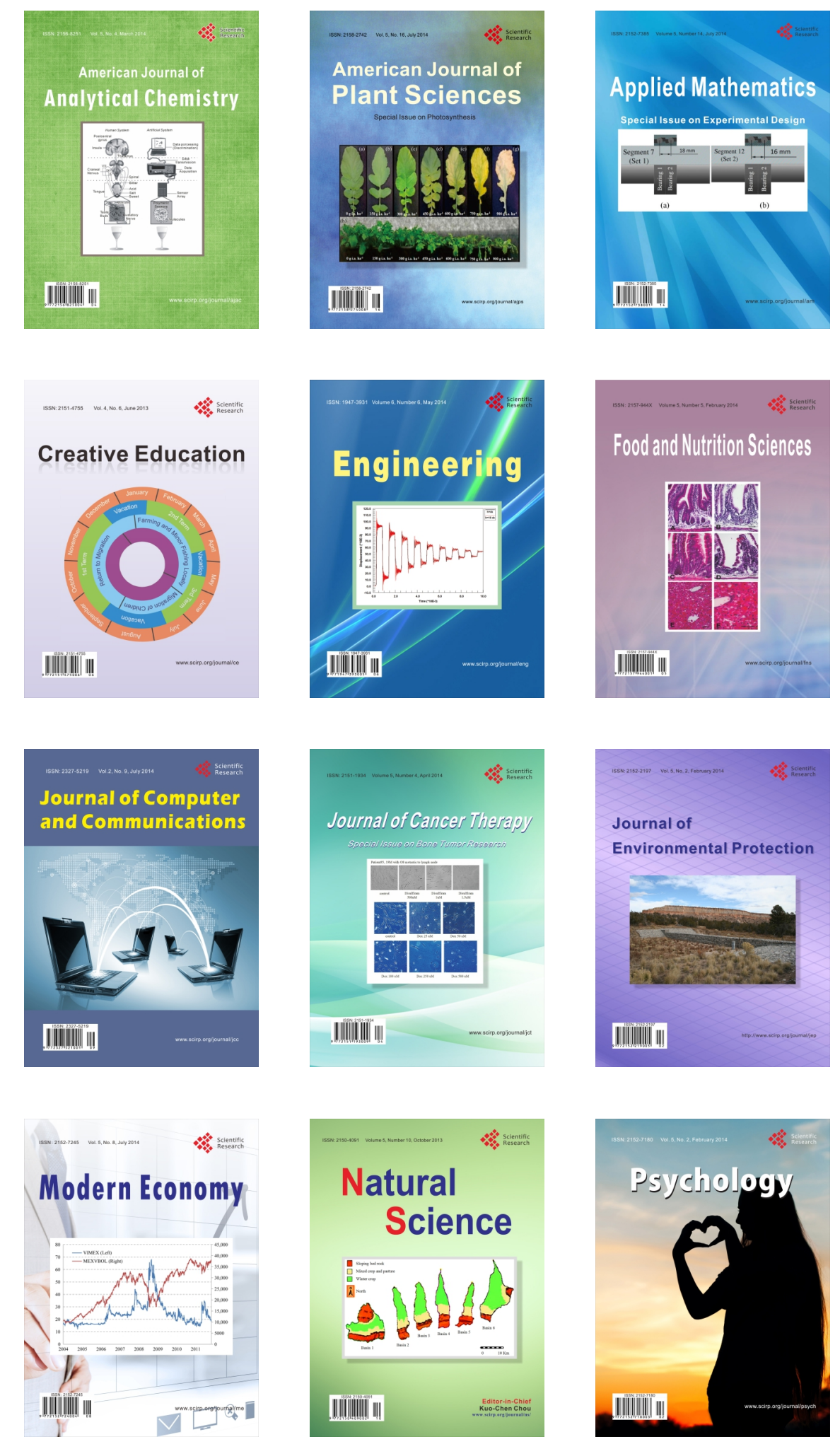